\title{
A Noninvasive Method to Measure Splanchnic Glucose Uptake after Oral Glucose Administration
}

Bernhard Ludvik, * John J. Nolan, * Anne Roberts, ${ }^{*}$ Joseph Baloga, * Mary Joyce, ${ }^{*}$ Jo M. Bell, * and Jerrold M. Olefsky From the ${ }^{*}$ Departments of Medicine and ${ }^{\ddagger}$ Radiology, University of California, San Diego, La Jolla, California 92093; and Veterans Administration Medical Center, San Diego, California 92161

\begin{abstract}
We have developed a noninvasive method to estimate splanchnic glucose uptake (SGU) in humans (oral glucose clamp technique [OG-CLAMP]), which combines a hyperinsulinemic clamp with an oral glucose load (oral glucose tolerance test). We validated this method in 12 nondiabetic subjects using hepatic vein catheterization (HVC) during an oral glucose tolerance test. During HVC, splanchnic blood flow increased from $1,395 \pm 64$ to $1,935 \pm 109 \mathrm{ml} / \mathrm{min}$, returning to basal after $180 \mathrm{~min}$ and accounted for $\mathbf{4 5} \pm 7 \%$ of SGU in lean and $19 \pm 5 \%$ in obese subjects $(P<0.05)$. SGU estimated during the OG-CLAMP was $22 \pm 2 \%$ of the glucose load, and this was significantly correlated $(r=0.90$, $P<0.0001)$ with SGU $(35 \pm 4 \%)$ and with first pass SGU $(24 \pm 3 \% ; r=0.83, P<0.001)$ measured during HVC. SGU was higher in obese than in lean subjects during OGCLAMP $(27 \pm 1 \%$ vs $18 \pm 3 \%, P<0.01)$ and HVC $(44 \pm 4 \%$ vs $26 \pm 5 \%, P<0.05$ ). In conclusion, SGU during the OGCLAMP is well correlated to SGU measured during HVC. An increase in splanchnic blood flow is a major contributor to SGU in lean subjects. SGU is increased in obese subjects as measured by both methods. (J. Clin. Invest. 1995. 95:2232-2238.) Key words: oral glucose tolerance test • clamp - hepatic vein catheter technique - splanchnic blood flow
\end{abstract}

\section{Introduction}

The liver plays a central role in the maintenance of normal glucose tolerance. After glucose ingestion the liver switches from glucose production to glucose uptake (1). Glucose uptake by the splanchnic area is regulated by the total amount of glucose presented to the splanchnic area and by its intrinsic ability to extract glucose (2). It has been shown that substantial glucose uptake occurs after ingestion or intraduodenal infusion of glucose

Address correspondence to Jerrold M. Olefsky, Department of Medicine, University of California, San Diego and Veterans Administration Medical Center (111G), 3350 La Jolla Village Drive, San Diego, CA 92161. Phone: 619-534-6651; FAX: 619-534-6653.

Received for publication 13 September 1994 and in revised form 10 January 1995.

1. Abbreviations used in this paper: dpm, disintegrations per minute GDR, glucose disposal rate; GINF, glucose infusion rate; HGO, hepatic glucose output; HVC, hepatic vein catheterization; OG-CLAMP, oral glucose clamp technique; OGL, oral glucose load; OGR, oral glucose resorption; $R_{\mathrm{d}}$, rate of glucose disappearance; SBF, splanchnic blood flow; SGU, splanchnic glucose uptake.

The Journal of Clinical Investigation, Inc.

Volume 95, May 1995, 2232-2238
(3). This is considered to be due to a negative arterial-portal glucose gradient, which exists during portal, but not peripheral glucose presentation. To assess splanchnic glucose uptake (SGU) ${ }^{1}$ and the disposition of an oral glucose load in humans, two methods - hepatic vein catheterization (HVC) and the double tracer approach - have been used. Hepatic vein catheterization allows measurement of net hepatic glucose output (HGO) and hepatic glucose uptake can be calculated by subtracting hepatic glucose output from the amount of glucose given (4). With this approach it has been concluded that up to $60 \%$ of an oral glucose load (OGL) is taken up by the liver (1).

Studies using the double tracer method revealed a much lesser amount of glucose retained by the liver, ranging from $9 \%(5)$ to $\sim 30 \%$ (6-8). Both methods have some limitations. Hepatic vein catheterization measures the net appearance of glucose on the systemic side of the liver, which is the amount of glucose transversing the liver after absorption from the gut, plus the remaining endogenous glucose production, which is suppressed by $\sim 50 \%$ after an oral glucose load (9). Potential complications resulting from the invasive nature of this procedure, such as local hematomas at the puncture site, infections and thrombosis, and radiation exposure due to fluoroscopy prevent this method from being used in repetitive experiments. Results obtained by the double tracer method may be affected by incorporation of the glucose tracers into glycogen, which may lead to an inaccurate estimation of the rate of appearance (6).

Limitations in the availability and in the repetitive use of both methods due to invasive procedures or radiation exposure have led us to evaluate a new, noninvasive approach to measure SGU by the combination of an euglycemic, hyperinsulinemic clamp and an oral glucose tolerance test (OGTT) during steady state of glucose disposal (oral glucose clamp [OG-CLAMP] method). This method could circumvent some of the problems with the methods mentioned above. To validate this method and to ensure that the different hormonal levels and secretion patterns do not influence measurement of SGU, we compared this method with the hepatic vein catheter technique in 12 nondiabetic subjects.

\section{Methods}

Subjects. 12 male, nondiabetic subjects participated in the study. Their ages ranged from 32 to $63 \mathrm{yr}$ (mean $44.7 \pm 2.4 \mathrm{yr}$ ), their body mass index (BMI) from 24.0 to $38.5 \mathrm{~kg} / \mathrm{M}^{2}$ (mean $30.9 \pm 1.5 \mathrm{~kg} / \mathrm{M}^{2}$ ). These subjects were further subdivided into six lean (BMI $26.5 \pm 0.9 \mathrm{~kg} / \mathrm{M}^{2}$ ) and six obese (BMI $35.3 \pm 1.2 \mathrm{~kg} / \mathrm{M}^{2}, P<0.001$, obese vs lean) subjects, matched for age $(44.7 \pm 4.4 \mathrm{vs} 44.7 \pm 2.4 \mathrm{yr})$. All participants were admitted $3 \mathrm{~d}$ before the respective study to the San Diego Veterans Administration Medical Center Special Diagnostic and Therapeutic Unit, and consumed a weight maintenance diet containing 55\% carbohydrate, $30 \%$ fat, and $15 \%$ protein. No subject had a positive family history for diabetes or was taking any medication known to affect glucose metabolism. The purpose, nature, and potential risks of the study were explained in detail to all subjects before obtaining their written consent. 
The study protocol was reviewed and approved by the Human Subjects Committee of the University of California, San Diego.

Experimental protocol. The order of the protocol was randomized. All studies were performed at 8:00 a.m. after a 10-12-h overnight fast. There was at least an interval of $1 \mathrm{wk}$ between the OG-CLAMP and the hepatic vein catheterization study.

\section{OG-CLAMP method}

Glucose clamp study. The glucose clamp was performed as described previously under euglycemic conditions to quantitatively measure glucose uptake and maintain plasma glucose and serum insulin levels at the required levels $(10,11)$. Using this approach, an antecubital vein was cannulated in an antegrade manner to administer infusates. A dorsal hand vein was cannulated in a retrograde fashion and kept in a warming device $\left(72^{\circ} \mathrm{C}\right)$ to facilitate venous sampling and provide arterialized venous blood. A loading dose of insulin was administered in a logarithmically decreasing manner over the next 10-min period and was followed immediately by a constant infusion rate $\left(120 \mathrm{mU} / \mathrm{M}^{2}\right.$ per min, for the next $420 \mathrm{~min}$ ). The plasma glucose was maintained at the desired level throughout the study by monitoring the glucose level at 5-min intervals and adjusting the infusion rate of a $20 \%$ dextrose solution using a servo-control negative feedback principle. Thus, plasma glucose and insulin level were kept constant while the glucose infusion varied and the rate of glucose uptake assessed by the concomitant administration of 3- $\left[{ }^{3} \mathrm{H}\right]$ glucose served as a direct measurement of insulin effectiveness.

Splanchnic glucose uptake study. After $3 \mathrm{~h}$ of the insulin infusion, the rate of glucose disposal was at a steady state and significantly stimulated above basal values, and hepatic glucose production was suppressed. At this point a 75-g oral glucose load, which was not isotopically labeled, was administered to the subjects over $1 \mathrm{~min}$ and the plasma glucose was maintained at the previous steady state level by adjustment of the rate of glucose infusion to compensate for gastrointestinal glucose absorption. Completion of absorption was indicated when the steady state glucose infusion set was reached again. The rate of splanchnic glucose uptake was then calculated by subtracting the decrease in the amount of glucose infused from the known amount of glucose ingested. Blood was sampled from the dorsal hand vein before the clamp, at steady state and every $15 \mathrm{~min}$ after administration of the oral glucose load to determine insulin, glucagon and C-peptide levels.

Measurement of $H G O$. Glucose turnover was quantitated by infusion of 3- $\left[{ }^{3} \mathrm{H}\right]$ glucose in a continuous fashion at a rate of $0.15 \mu \mathrm{Ci} / \mathrm{min}$. 3$\left[{ }^{3} \mathrm{H}\right]$ glucose was infused for a 4-h infusion period preceding all measurements. For measurement of basal hepatic glucose production rate, samples were obtained at 10 -min intervals for a subsequent $30 \mathrm{~min}$. During the clamp study, measurements were made at 20-min intervals to determine both the concentration and specific activity. Changes in glucose turnover were calculated using the variable tracer hot-glucose infusion rate (hot-GINF) infusion technique (12), which allows calculation of glucose disposal during non-steady state.

Evaluation of stability of glucose disposal rate after OGL. To investigate whether the glucose disposal rate measured during steady state changes after glucose administration, the OG-CLAMP was repeated in six subjects with a continuous infusion of $3-\left[{ }^{3} \mathrm{H}\right]$ glucose at a rate of 0.6 $\mu \mathrm{Ci} / \mathrm{min}$ without labeling the infusate. At basal and at 20-min intervals throughout the experiment, samples were obtained for determination of glucose concentration and specific activity. As the peripheral glucose infusion and the oral glucose load were not labeled and the glucose level was kept at the same level as before oral glucose administration, . a change in specific activity would indicate a change in glucose disposal rate.

HVC study. Under local anesthesia with $2 \%$ lidocaine, the femoral artery was punctured with an 18-gauge needle and a 5 French Teflon catheter was introduced. The catheter tip was positioned fluoroscopically at the level of the inferior margin of the sacroiliac joint so that it remained distal to the origin of the internal iliac artery. The femoral vein was similarly punctured, and a 6.5 French polyethylene catheter was advanced via the inferior vena cava into the right-sided hepatic vein under fluoroscopic control. The catheter was advanced into a wedge position and then withdrawn $1-2 \mathrm{~cm} .1 \mathrm{ml}$ of contrast medium was injected to visualize the tip of the catheter and to ensure that it was positioned in an area of adequate blood flow.

Splanchnic blood flow was estimated by a primed continuous infusion of indocyanine green (13). The dye infusion was started via an antecubital vein $75 \mathrm{~min}$ before glucose ingestion and continued throughout the study. Blood was sampled simultaneously from the artery and the hepatic vein at 10 -min intervals starting $45 \mathrm{~min}$ after the beginning of green dye infusion. At time zero, the subjects ingested $300 \mathrm{ml}$ of a 75-g glucose solution over $5 \mathrm{~min}$. Arterial and hepatic venous blood was sampled at 15 -min intervals to determine the concentrations of glucose, C-peptide, insulin, glucagon, and indocyanine green for $4 \mathrm{~h}$ after glucose ingestion.

Splanchnic plasma flow was calculated by dividing the green dye infusion rate by arteriohepatic venous dye concentration difference. Splanchnic blood flow was estimated by dividing splanchnic plasma flow by ( 1 - hematocrit).

\section{Calculations}

OG-CLAMP method. GINF ( $\mathrm{mg} / \mathrm{kg}$ per $\mathrm{min}$ ) was calculated for 20 min intervals and corrected for changes in pool fraction throughout the entire experiment. Activation of glucose disposal rate (GDR) and the time to steady state is faster the higher the insulin level, and in most subjects, GINF was constant between 120 and 180 min, and the mean value for GINF between 120 and $180 \mathrm{~min}$ was taken as the steady state for calculation. We have previously shown that in insulin-resistant obese subjects, activation is slower than in controls (14), and, therefore, in some of the obese subjects GINF rose slightly between 120 and 180 minutes. In these subjects, the clamp was extended for an additional 40-60 min until steady state was reached, before administration of the oral glucose load. By this means, we confirmed that there was no further rise in GINF after $160 \mathrm{~min}$, and used the GINF between 160 and 180 min for the steady state calculation. The validity of this approach was substantiated by the fact that the GINF returned to this same level after completion of absorption of the oral glucose load. Steady state conditions were further confirmed by the demonstration of stable plasma glucose specific activity in the cold-GINF experiment (see Fig. 3 ). After the oral glucose administration the respective GINF rates were subtracted from the steady state GINF. Multiplication of the difference in GINF with the respective time interval used for calculation ( $20 \mathrm{~min}$ ) and the body weight, yields the absolute reduction of GINF in grams of glucose. The integrated reduction of GINF over the time required for glucose absorption was then subtracted from the known amount of glucose given $(75 \mathrm{~g}$ ). SGU was expressed as percentage of the administered glucose load retained by the splanchnic area.

HVC method. SGU during HVC was calculated by a modified formula reported by Ferrannini et al. (15). The hepatic vein catheter technique allows measurement of the splanchnic blood flow (SBF), the glucose concentration in the artery (Ga) and in the hepatic vein (Ghv). In the fasting state, the splanchnic bed receives glucose via the hepatic and mesenteric arteries at the systemic glucose concentration; the glucose influx into the splanchnic area is therefore SBF $\cdot \mathrm{Ga}$. After administration of an OGL, the portal glucose level rises and the total glucose influx to the splanchnic area is then $\mathrm{SBF} \cdot \mathrm{Ga}$ plus the rate of oral glucose resorption (OGR). The glucose efflux from the splanchnic bed is Ghv $\cdot$ SBF and must equal the sum of the total glucose influx after extraction. The relation is shown by the following equation:

$\mathrm{SBF} \cdot \mathrm{Ghv}=(\mathrm{SBF} \cdot \mathrm{Ga}+\mathrm{OGR})(1-\mathrm{F})$

where $\mathrm{F}$ is the fractional extraction of glucose. Solving the equation for $F$, one obtains:

$F=\frac{S B F \cdot G a+O G R-S B F \cdot G h v}{S B F \cdot G a+O G R}$

Lacking a method to measure portal glucose levels, the only unknown in this equation is OGR. However, assuming complete absorption of the glucose load, OGR can be substituted by the oral glucose load. Completion of absorption of the glucose load can be assumed when the 
fasting hepatic glucose output is reached again, and the time required for absorption of the glucose load $\mathrm{T}$ ( $\mathrm{abs}$ ) can then be used for calculating the integrated hepatic fractional extraction according to the following formula:

$F=\frac{O \int^{T(a b s)} \operatorname{SBF}(t) G a(t) d t+O G L-O \int^{T(a b s)} \operatorname{SBF}(t) G h v(t) d t}{O \int^{T(a b s)} \operatorname{SBF}(t) G a(t) d t+O G L}$

The numerator in equation 2 represents the total amount of glucose taken up by the splanchnic area per unit time (SG). Rearranging equation 2 , one obtains:

$S G=F \cdot O \int^{T(a b s)} S B F(t) G a(t) d t+O G L$

The relative amount of glucose taken up by the splanchnic area after an oral glucose load (SGU) can be expressed as:

$\operatorname{SGU}(\%)=\frac{S G}{\mathrm{OGL}} \cdot 100$

The HVC technique measures the net appearance of glucose on the systemic side and, therefore, includes glucose that has already passed through the splanchnic area and has not been taken up by peripheral tissues. During the OG-CLAMP method, an increase in the arterial glucose level is prevented by reducing the GINF to compensate for gastrointestinal glucose absorption. The OG-CLAMP method, therefore, measures first pass SGU, whereas the HVC method measures the integrated SGU over the period of glucose absorption. As the fractional hepatic glucose extraction does not change during peripheral hyperglycemic conditions comparable to those during the HVC experiment in our patients $(15,16)$, it is valid to calculate first pass SGU by substituting $\mathrm{Ga}$ in equation 4 by the basal arterial glucose level (Gabas). The total amount of glucose taken up by the splanchnic area per unit time after the first pass (SGfp) becomes then:

$S G f p=F \cdot\left(O \int^{T(\text { abs })} \operatorname{SBF}(t) \cdot G a b a s(t) d t+O G L\right)$

The relative amount of glucose taken up by the splanchnic area after the first pass (SGUfp) can then be calculated according to the formula:

$\operatorname{SGUfp}(\%)=\frac{\text { SGfp }}{\text { OGL }} \cdot 100$

These equations do not account for residual hepatic glucose production, which has been shown to decrease to $\sim 40-50 \%$ of its fasting value (8). Consequently, the weight of this term becomes very small relative to the glucose influx, and has been assumed to be zero. If a definitive value is assigned to it, the values for $\mathrm{F}$ and SGU will become slightly higher. This, however, applies to lean and obese subjects to the same extent and therefore does not change the correlation between the two methods or the comparison between the respective groups.

Portal insulin levels were derived from arterial and hepatic vein Cpeptide levels assuming an equimolar release of C-peptide and insulin by the beta cell (17), and neglecting the minimal C-peptide retention by the liver (18) according to the equation:

INSULIN $_{\text {portal venous }}$

$$
=\text { INSULIN }_{\text {arerial }}+\left(\text { C-PEPTIDE } E_{\text {hepatic venous }}-\text { C-PEPTIDE } E_{\text {arerial })}\right.
$$

Hepatic insulin extraction was calculated by subtracting the areas under the curve for portal insulin from that for hepatic vein insulin and expressed in relation to portal insulin output.

Measurements. Glucose was measured with an automated analyzer (Yellow Spring Instrument Co., Yellow Springs, $\mathrm{OH}$ ). Insulin was assayed by a double antibody radioimmunoassay according to the method of Desbuquois and Aurbach (19). C-peptide (20) and glucagon (21) were measured as described previously. Indocyanine green was analyzed by spectrophotometer after precipitation with sodium-desoxycholate (22).

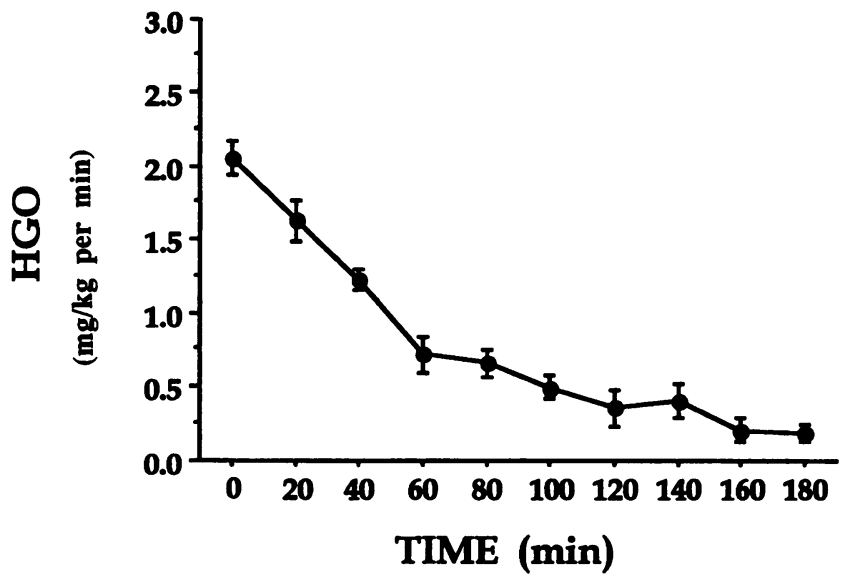

Figure 1. Endogenous HGO during the first part of the OG-CLAMP before the administration of oral glucose.

Statistical analysis. All data are presented as mean \pm SEM. All statistical comparisons between different groups were performed by the unpaired $t$ test analysis. Changes from baseline within any given group were evaluated by the paired $t$ test. Coefficients of correlation were determined with the Stat View program (Abacus Concepts, Inc., Berkeley, CA).

\section{Results}

Euglycemic glucose clamp + oral glucose load (OG-CLAMP) technique. With the OG-CLAMP method, insulin is infused at a rate of $120 \mathrm{mU} / \mathrm{M}^{2}$ per min for $3 \mathrm{~h}$ under euglycemic glucose clamp conditions until steady state rate of disappearance $\left(\boldsymbol{R}_{\mathrm{d}}\right)$ is achieved. At this time, basal HGO was suppressed by $91 \%$ from $2.06 \pm 0.12$ to $0.19 \pm 0.06 \mathrm{mg} / \mathrm{kg}$ per $\mathrm{min}$ ) (Fig. 1) and GDR increased to a steady state value of $7.19 \pm 0.51 \mathrm{mg} / \mathrm{kg}$ per min. Half maximal suppression of HGO occurred at $58 \pm 17 \mathrm{~min}$ and half maximal stimulation of $R_{\mathrm{d}}$ at $30 \pm 10 \mathrm{~min}$. Under these conditions in which HGO was essentially completely suppressed, the exogenous GINF approximates $\boldsymbol{R}_{\mathrm{d}}$. As seen in Fig. 2 , GINF gradually rises during the insulin infusion, reaching a value of $7.19 \pm 0.51 \mathrm{mg} / \mathrm{kg}$ per min at $180 \mathrm{~min}$. At this time, the 75-g oral glucose load was administered. Providing $\boldsymbol{R}_{\mathrm{d}}$ remains unchanged (see Fig. 3) any absorbed glucose which bypasses the liver to enter the peripheral circulation will increase the plasma glucose level unless GINF is decreased by an exact amount so as to maintain constant delivery of glucose to the peripheral circulation. As shown in Fig. 2 A, within 10-20 min after oral glucose administration it was necessary to decrease GINF to maintain plasma glucose levels constant (Fig. $2 \mathrm{~B}$ ). GINF was gradually decreased to a nadir of $1.74 \pm 0.45 \mathrm{mg} / \mathrm{kg}$ per min at $60 \mathrm{~min}$, reflecting posthepatic delivery of glucose from the gastrointestinal tract. After this time, GINF was gradually increased until the preloading rate of glucose infusion was reached 3-4 $\mathrm{h}$ after oral glucose administration. The rise in GINF reflects the decreasing influx of orally derived glucose, and the return of GINF to the preloading rate marks the end of the period of glucose absorption. The mean $\pm S E$ time at which GINF returned to the original rate was $163 \pm 9 \mathrm{~min}$. Plasma glucose levels were relatively stable throughout the 4-h postoral glucose loading period (Fig. $2 B$ ), indicating that the decrease in exogenous GINF was well matched to the posthepatic delivery rate of the orally ingested glucose. With this approach, the 

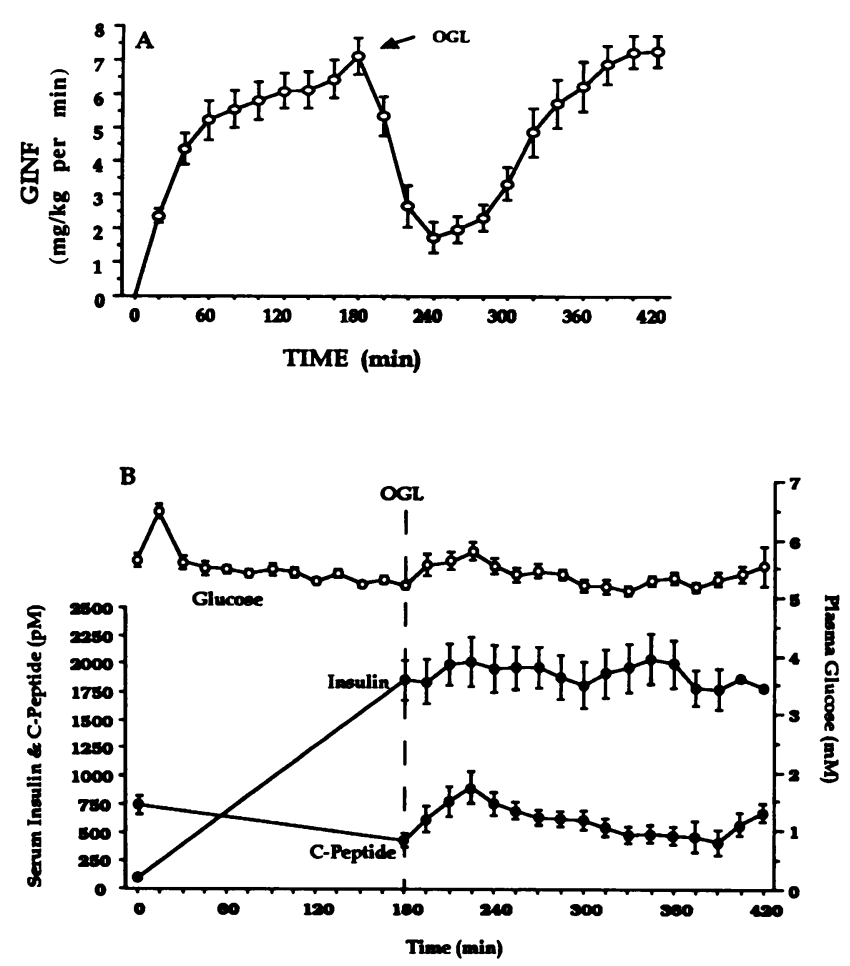

Figure 2. (A) GINF during the OG-CLAMP. (B) Plasma glucose (top), serum insulin (center), and C-peptide levels (bottom) during the OGCLAMP.

integrated decrease in the GINF over this 4-h period, subtracted from the amount of oral glucose given $(75 \mathrm{~g})$, is equal to the amount of oral glucose retained by the splanchnic bed, i.e., first pass splanchnic glucose uptake (see Methods).

During the $4 \mathrm{~h}$ after administration of oral glucose, the circulating insulin levels remained constant (Fig. 2 B). C-peptide levels rose over the first $30 \mathrm{~min}$ after oral glucose, but this effect on insulin secretion was small in comparison to the exogenous insulin infusion rates and did not appreciably alter the serum insulin concentrations (Fig. $2 B$ ). Serum glucagon levels decreased from a basal value of $125.7 \pm 14.0 \mathrm{ng} /$ liter to

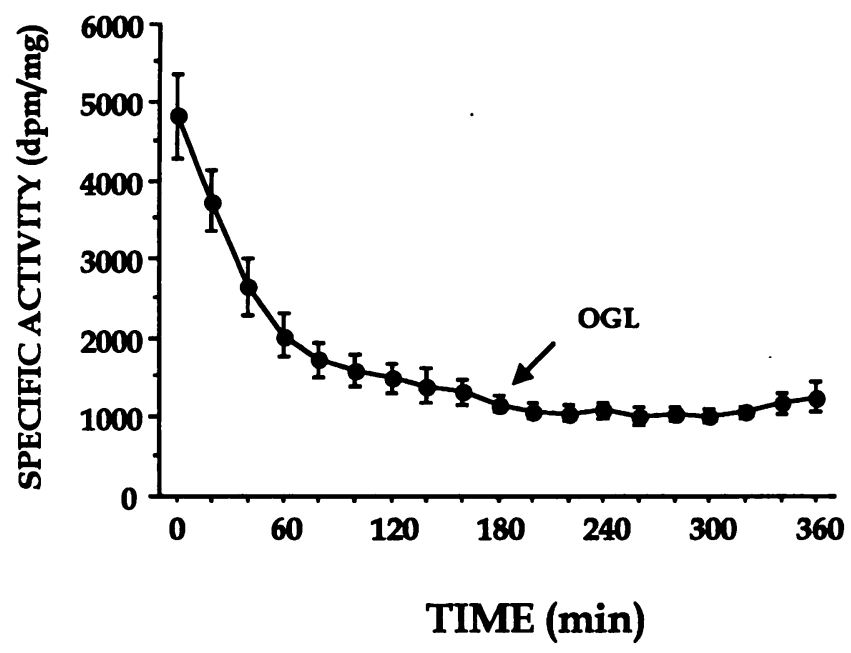

Figure 3. Plasma glucose specific activity $(\mathrm{dpm} / \mathrm{mg})$ during the OGCLAMP experiment.

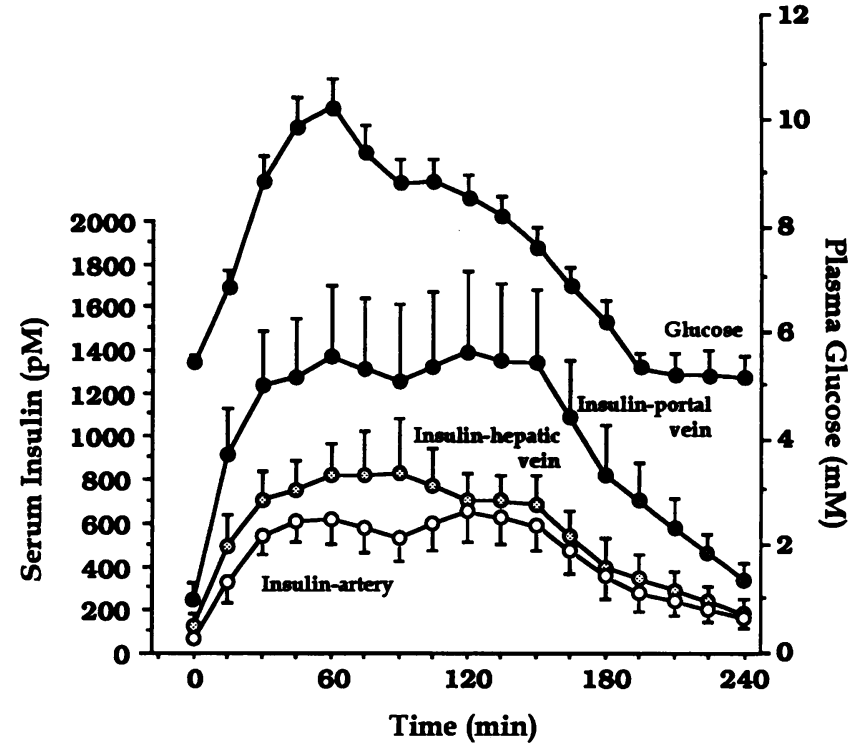

Figure 4. Arterial plasma glucose concentrations and insulin concentrations in the artery, hepatic vein, and those calculated for the portal vein, during the HVC experiment.

$93.3 \pm 9.4 \mathrm{ng} /$ liter at steady state $(P<0.0001)$, and did not change following oral glucose administration.

As shown in Fig. $1, R_{\mathrm{d}}$ rises and HGO is suppressed by $>90 \%$, as measured with the variable tracer infusion (hot-GINF) method. To determine whether the steady state disposal rate, achieved at the end of the glucose clamp period, is altered after oral glucose administration, OG-CLAMP studies were repeated in six subjects using a continuous infusion of $3-\left[{ }^{3} \mathrm{H}\right]$ glucose at a rate of $0.6 \mu \mathrm{Ci} / \mathrm{min}$. Since neither the peripheral glucose infusion, nor the oral glucose load were labeled in these studies, and since the circulating glucose level was kept constant before and during oral glucose administration, a change in glucose disposal rate after oral glucose would result in a corresponding change in glucose specific activity. As seen in Fig. 3, after initiation of the insulin infusion, glucose specific activity decreased from $4,774 \pm 628$ disintegrations per minute $(\mathrm{dpm}) / \mathrm{mg}$ to $1,170 \pm 95$ $\mathrm{dpm} / \mathrm{mg}$ at the end of the glucose clamp phase, and following oral glucose administration, specific activity remained constant over the next $3 \mathrm{~h}$. This shows that the glucose disposal rate was not altered by the oral glucose load, indicating that it is valid to extrapolate the steady state GDR to calculate SGU.

From these experiments we calculated that the cumulative hepatic glucose output during the $4 \mathrm{~h}$ after the oral glucose load was $58.2 \pm 1.5 \mathrm{~g}$. This means that splanchnic glucose uptake of the $75-\mathrm{g}$ glucose load was $22.4 \pm 2.0 \%$.

Hepatic vein catheterization. Fig. 4 presents the mean glucose and insulin levels over the 4-h period after administration of the 75-g glucose load during the HVC study. As can be seen, arterial glucose values rose and then returned to baseline by $180 \mathrm{~min}$. Arterial and hepatic vein insulin levels, as well as the calculated portal vein insulin levels (see Methods), are also shown in Fig. 4. The area under the curve for insulin secretion over the $180 \mathrm{~min}$ was significantly higher in the portal vein (219 $\mu \mathrm{M} / 3 \mathrm{~h}$ ) compared to the hepatic vein $(122 \mu \mathrm{M} / 3 \mathrm{~h})$, yielding an hepatic insulin extraction ratio of $40.4 \pm 3.6 \%$. After oral glucose administration, the splanchnic blood flow increased from $1,395 \pm 64 \mathrm{ml} / \mathrm{min}$ at basal to a peak of $1,935 \pm 109 \mathrm{ml} /$ min after $30 \mathrm{~min}$, and then declined to $1,437 \pm 122 \mathrm{ml} / \mathrm{min}$ at 

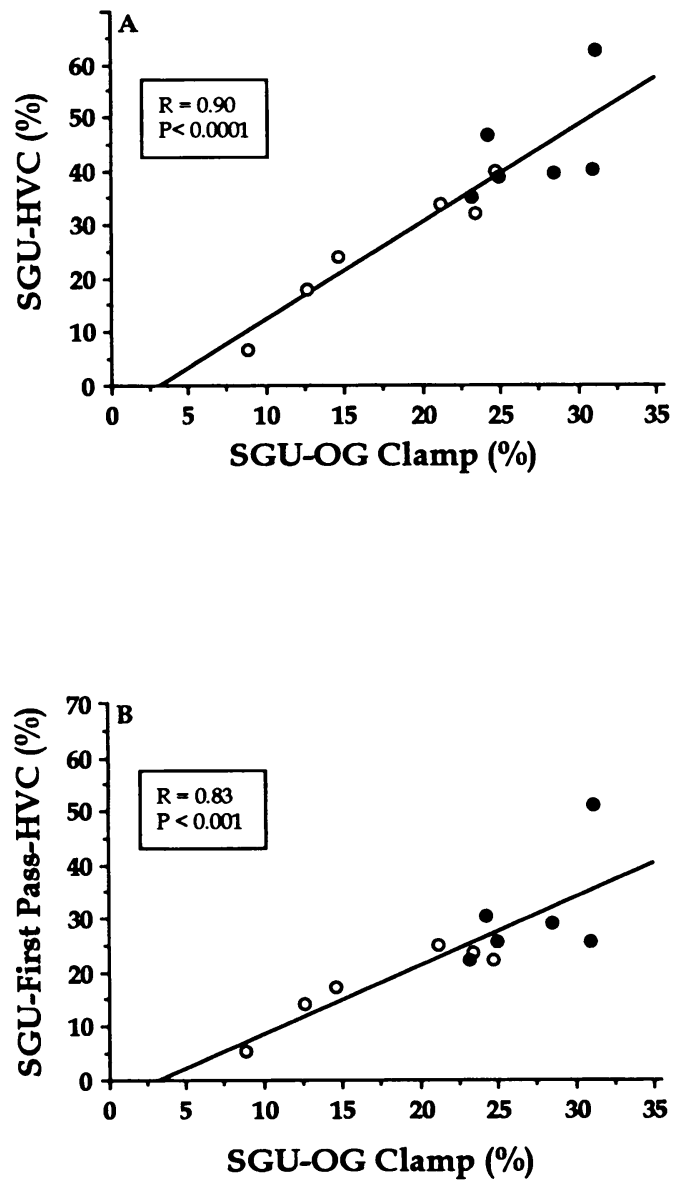

Figure 5. (A) Correlation between SGU during the OG-CLAMP and HVC. $(B)$ Correlation between SGU during the OG-CLAMP and first pass SGU during HVC. (open circles) lean subjects; (closed circles) obese subjects.

$180 \mathrm{~min}$. Posthepatic glucose delivery rose from a basal value of $133.4 \pm 7.7 \mathrm{mg} / \mathrm{min}$ to a peak of $544.7 \pm 65.4 \mathrm{mg} / \mathrm{min}$ at 45 min and then declined to $173.8 \pm 60.1 \mathrm{mg} / \mathrm{min}$ at $180 \mathrm{~min}(P$ $=0.53$ vs basal). Completion of glucose absorption was determined as the time when hepatic glucose delivery had returned to the basal value. The mean time required for glucose absorption was $168 \pm 11 \mathrm{~min}$. Serum glucagon levels (125.7 $\pm 14.3 \mathrm{ng} /$ liter at basal) were significantly suppressed during the OGL $(100.1 \pm 14.3 \mathrm{ng} /$ liter at $120 \mathrm{~min}, 106.0 \pm 13.5 \mathrm{ng} /$ liter at 180 $\min , P<0.05)$.

During the HVC study cumulative posthepatic glucose delivery can be calculated as described in the Methods section. With this approach $48.9 \pm 3.7 \mathrm{~g}$ of glucose were delivered to the peripheral circulation after oral glucose loading. Thus, SGU was $34.8 \pm 4.1 \%$ of the 75 -g glucose load. First pass SGU was calculated as $24.3 \pm 3.1 \%$.

Comparison between OG-CLAMP and HVC. Both methods of assessing SGU yielded comparable absolute values when all subjects were considered as a group. To more thoroughly compare these methods, the relationship between individual values was examined. As shown in Fig. 5, a highly significant correlation was observed between SGU, as measured during the OGCLAMP, and SGU ( $r=0.90, P<0.0001)$, or first pass SGU ( $r=0.83, P<0.001)$ as measured by HVC. The correlation for SGU between the OG-CLAMP and the HVC method remained

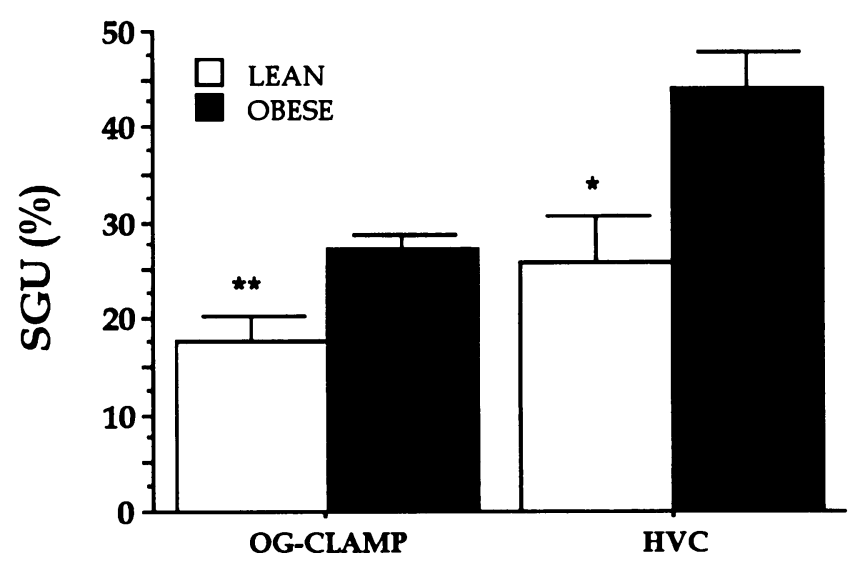

Figure 6. SGU in lean and obese subjects measured by the OG-CLAMP method and HVC. $* P<0.05 ; * * P<0.01$.

highly significant when a residual endogenous hepatic glucose production of $50 \%$ of basal was assumed during the HVC experiment $(r=0.86, P<0.001)$.

Comparison between lean and obese subjects. The obese subjects were clearly insulin resistant, exhibiting a significantly lower GDR ( $5.78 \pm 0.4 \mathrm{mg} / \mathrm{kg}$ per $\mathrm{min})$ than the control subjects $(8.61 \pm 0.43 \mathrm{mg} / \mathrm{kg}$ per $\mathrm{min}, P<0.001)$. The time required for resorption of glucose during the OG-CLAMP was not different between lean and obese subjects $(157 \pm 39 \mathrm{~min}$ vs $170 \pm 21 \mathrm{~min}$, $P=0.48)$. As indicated in Fig. 5, the individual values for SGU were higher in the obese subjects compared to the controls by both HVC and OG-CLAMP methods, and these data are summarized in Fig. 6 . In the obese subjects posthepatic delivery of glucose was significantly lower compared to lean subjects during either the OG-CLAMP $(54.5 \pm 1.1$ vs $61.8 \pm 2.0 \mathrm{~g}, P$ $<0.01)$ or the HVC $(42.1 \pm 3.0$ vs $55.7 \pm 3.7 \mathrm{~g}, P<0.05)$. SGU was significantly higher in obese compared to lean subjects during OG-CLAMP $(27.2 \pm 1.4 \%$ vs $17.6 \pm 2.6 \%, P$ $<0.01)$ as well as during $\mathrm{HVC}(43.9 \pm 4.0 \%$ vs $25.8 \pm 4.9 \%, P$ $<0.05)$. This difference remained significant when a residual endogenous hepatic glucose production of $50 \%$ of basal was assumed during the HVC experiment $(P<0.05)$.

\section{Discussion}

The regulation of hepatic glucose uptake after glucose ingestion is determined by the hormonal milieu of insulin and glucagon, the glucose load to the liver, and the route of glucose delivery $(3,23)$. In animal studies it has been shown that hepatic glucose uptake after oral glucose administration accounts for $30 \%$ of the glucose load $(24,25)$. As the portal vein is not routinely accessible to cannulation in humans, it is not possible to measure the amount of glucose retained by the liver without including the component of extra hepatic splanchnic glucose consumption. The results obtained in humans, therefore, represent SGU. Currently, there are two experimental methods used to assess SGU in man: $(a) \mathrm{HVC}$, and $(b)$ the double-tracer method, in which oral and circulating glucose are traced with separate radioactive glucose isotopes. In the current studies, we have developed a third method, termed the OG-CLAMP to assess SGU in humans, and have applied this new method to quantitation of SGU in lean controls and insulin-resistant obese subjects.

The HVC technique requires catheterization of an artery 
and the hepatic vein, which allows measurement of the net balance of glucose across the splanchnic bed after glucose administration (4). This method, however, cannot separate out continuing endogenous glucose production by the liver, as a component of posthepatic glucose delivery, and, therefore, may underestimate SGU (8). To calculate SGU, it is necessary to determine the time required for complete absorption of the oral glucose load. This is indicated by the return of HGO and splanchnic blood flow to baseline values. Studies using isotopes have produced somewhat different results regarding the time period required for complete absorption of an oral glucose load, ranging from 120 to $240 \mathrm{~min}$ (26 and 5, respectively); most investigators, however, assume complete absorption after 180 min. Other disadvantages of the HVC technique are the invasive nature of the procedure and the expertise required for catheter placement. These limitations restrict the use of HVC, particularly in sick patients, or when repeated measurements of SGU are necessary.

The double-tracer technique uses an intravenous infusion of labeled glucose to trace total rate of appearance, and a second isotope of glucose added to the ingested glucose load to trace the contribution of absorbed glucose to total rate of appearance (27). However, concurrent glycogenesis and glycogenolysis and/or glucose/glucose-6-phosphate cycling may lead to an over- or underestimation of SGU, depending on the isotopes used (6). Like the HVC technique, this method also cannot accurately indicate completion of oral glucose absorption.

The OG-CLAMP method provides a relatively noninvasive measurement of SGU and is based on the combination of a euglycemic, hyperinsulinemic clamp and oral glucose administration. In this manner, a hyperinsulinemic euglycemic clamp is carried out for $3 \mathrm{~h}$ to establish a state of HGO suppression and steady state GDR; this is followed by ingestion of oral glucose and continuation of the glucose clamp for the subsequent $4 \mathrm{~h}$. The following assumptions underly this method, and, therefore require validation: (a) HGO must be suppressed, and GDR must be at steady state before oral glucose administration. (b) The GDR must remain at steady state throughout the period of SGU measurement. (c) Since the plasma insulin level and/ or the insulin/glucagon ratio may influence SGU, an effect of the hyperinsulinemia and/or the high insulin/glucagon ratio during the OG-CLAMP on SGU must be negligible.

Each of the above assumptions has been verified by the results presented. Thus, using the variable tracer infusion (hotGINF) method (12), we demonstrated that GDR reached steady state and endogenous HGO was suppressed by $91 \%$ after 180 min of the hyperinsulinemic clamp. In a subset of subjects, an additional OG-CLAMP was performed using a continuous 3$\left[{ }^{3} \mathrm{H}\right]$ glucose infusion, without labeling the glucose infusate. Since the plasma glucose level (and, therefore, the glucose pool) was kept constant throughout the study, any change in GDR would lead to a change in specific activity of the 3$\left[{ }^{3} \mathrm{H}\right]$ glucose tracer. As shown in Fig. 3, no change in specific activity occurred throughout the period of glucose absorption and, therefore, GDR remained constant throughout this period. Therefore, one can extrapolate the steady state GDR after 180 min. To suppress HGO, we had to infuse insulin at a rate of $120 \mathrm{mU} / \mathrm{M}^{2}$ per min yielding supra physiologic insulin levels of $\sim 1,800 \mathrm{pM}$. Had we used a lower insulin infusion rate, then it would have taken longer to reach steady state GDR, and HGO would not have been completely suppressed, particularly in insulin-resistant subjects. Furthermore, with this approach, GDR must be high enough to allow an appropriate reduction in GINF to compensate for gastrointestinal glucose absorption and still maintain euglycemia. However, this method of insulin delivery leads to peripheral insulin concentrations during the OGCLAMP which were threefold higher than those measured in the hepatic vein and 1.5 times higher than those calculated in the portal vein during HVC. On the other hand, glucagon levels were the same during the OG-CLAMP and HVC studies. Most importantly, however, examination of the results from the two methods demonstrates that the calculated SGU values are comparable and well correlated. Since these two approaches are quite independent of each other, and since the HVC technique provides a well accepted method of SGU estimation, these data argue strongly that the supraphysiologic insulinemia during the OG-CLAMP did not measurably influence SGU. This conclusion is in good agreement with previous findings in humans showing that neither hyperinsulinemia nor hypoglucagonemia independently enhanced SGU $(2,28,29)$. Furthermore, Myers et al. demonstrated in dogs that the dose-response relationship between insulin concentration and hepatic glucose uptake approaches saturation at portal insulin levels of $80 \mu \mathrm{U} / \mathrm{ml}$ (30). This might further explain why the supraphysiologic insulin levels during the OG-CLAMP do not lead to greater SGU than that measured during HVC.

Taken together, the above considerations indicate that SGU as measured during the OG-CLAMP method is comparable to the results obtained during HVC. The values for SGU are higher for the HVC technique than for the OG-CLAMP. This is because the HVC technique measures the net appearance of glucose on the systemic side and, therefore, includes glucose that has already passed through the splanchnic area and has not been taken up by the peripheral tissues. During the OG-CLAMP method, an increase in the arterial glucose concentration is prevented by reducing the GINF to compensate for gastrointestinal glucose absorption. The OG-CLAMP method, therefore, measures first pass SGU, whereas the HVC method measures integrated SGU over the period of glucose absorption. This explains why SGU values calculated from the HVC are greater than those calculated from the OG-CLAMP, which are very similar to those reported for the double tracer technique (31). However, when first pass SGU is calculated from the HVC experiment, the values are comparable to those calculated from the OGCLAMP. As such, the OG-CLAMP technique represents a relatively noninvasive approach allowing the measurement of GDR, HGO, and first pass SGU in a single experiment. The method can be conducted without a need for complex tracer measurements or kinetic analyses, and does not require the availability of angiographic facilities and hepatic catheter placement expertise. This could facilitate repeated measures of SGU in individual subjects after various metabolic perturbations and treatments. Furthermore, with this approach, SGU can be more conveniently assessed across a wider spectrum of pathophysiologic states.

In this latter regard, we have used both the OG-CLAMP and HVC approach to assess SGU in lean control, compared to insulin-resistant obese subjects. We found that the time required for completion of gastrointestinal glucose absorption was essentially the same when measured with the two techniques, and, more importantly, was comparable between the two study groups. Interestingly, when SGU was compared, a 30-40\% increase was observed in the obese subjects, and this was independently confirmed using both HVC and the OG-CLAMP approach. The mechanisms underlying this phenomenon are unclear at the present time. One might postulate that this is due 
to the increased splanchnic adipose tissue mass in the obese patients, leading to augmentation of the nonhepatic component of SGU. While our data do not directly disprove this notion, earlier studies have shown that nonhepatic SGU makes only a small contribution $(\sim 10 \%)$ to overall SGU (23). Furthermore, total body adipose tissue mass accounts for only a minor portion of overall body glucose disposal. Therefore, even though obese subjects have a greater splanchnic adipose tissue mass, it seems unlikely that this makes a major contribution to glucose extraction, accounting for the increased SGU in the obese subjects. It seems more likely, therefore, that the increased SGU in obesity is due to enhanced hepatic glucose uptake. In this event, the mechanisms and physiologic significance of this observation deserve further study. It should be noted that if SGU were not increased in obesity, then postprandial glycemic excursions would be greater. In this sense, increased SGU may protect against postprandial hyperglycemia in these insulin-resistant patients.

In summary, we have used a technique to estimate SGU in human subjects which we have termed the OG-CLAMP approach. The results provide data to support the assumptions underlying this technique, and we have validated it by showing quantitative comparability with measurements of SGU by the more accepted HVC approach. This approach should facilitate measurement of splanchnic glucose balance in various abnormal metabolic and pathophysiologic states, and should allow repeated measurements of SGU in individual subjects to assess the effects of various treatments and perturbations on splanchnic glucose balance. In these current studies, we have applied this technique to the examination of splanchnic glucose balance in obese insulin-resistant subjects and have found that SGU is significantly higher in obesity compared to controls. Increased SGU could be viewed as a means to protect peripheral tissues against unwanted postprandial hyperglycemia.

\section{Acknowledgments}

Bernhard Ludvik is a recipient of a Max-Kade Foundation Postdoctoral Fellowship Award and is on leave from the University of Vienna, Austria.

The work was supported by National Institutes of Health grants DK 33649, General Clinical Research Center grant RR00827 and Medical Service, Department of Veterans Affairs, Veterans Administration Medical Center, San Diego, CA.

\section{References}

1. Felig, P., J. Wahren, and R. Hendler. 1975. Influence of oral glucose ingestion on splanchnic glucose and gluconeogenic substrate metabolism in man Diabetes. 24:468-475.

2. DeFronzo, R. A. 1987. Regulation of hepatic glucose metabolism in humans. Diabetes Metab. Rev. 3:415-459.

3. Pagliasotti, M., and A. D. Cherrington. 1992. Regulation of net hepatic glucose uptake in vivo. Annu. Rev. Physiol. 54:847-860.

4. Felig, P., J. Wahren, and R. Hendler. 1978. Influence of maturity-onset diabetes on splanchnic glucose balance after oral glucose ingestion. Diabetes. 27:121-126.

5. Radziuk, J., T. J. McDonald, D. Rubenstein, and J. Dupre. 1978. Initial splanchnic extraction of ingested glucose in normal man. Metab. Clin. Exp. 27:657-669.

6. Butler, P. C., and R. A. Rizza. 1991. Contribution to postprandial hyperglycemia and effect on initial splanchnic glucose clearance of hepatic glucose cycling in glucose-intolerant or NIDDM patients. Diabetes. 40:73-81.
7. Kelley, D., A. Mitrakou, H. Marsh, F. Schwenk, J. Benn, G. Sonnenberg, M. Arcangeli, T. Aoki, J. Sorensen, M. Berger, et al. 1988. Skeletal muscle glycolysis, oxidation, and storage of an oral glucose load. J. Clin. Invest. 81:15631571.

8. Ferrannini, E., D. C. Simonson, L. D. Katz, G. Reichard, S. Bevilacqua, E. J. Barrett, M. Olsson, and R. A. DeFronzo. 1988. The disposal of an oral glucose load in patients with non-insulin-dependent diabetes. Metab. Clin. Exp. 37:79-85.

9. Ferrannini, E., O. Bjorkman, G. A. Reichard, A. Pilo, A. Olsson, J. Wahren, and $\mathbf{R}$. DeFronzo. 1985. The disposal of an oral glucose load in healthy subjects. Diabetes. 34:121-126.

10. Olefsky, J. 1981. Insulin resistance and insulin action: an in vitro and in vivo perspective. Diabetes. 30:148-162.

11. Olefsky, J. M., and O. G. Kolterman. 1981. Mechanisms of insulin resistance in obesity and non-insulin-dependent (type II) diabetes. Am. J. Med. 70:151-168.

12. Molina, J. M., A. D. Baron, S. V. Edelman, G. Brechtel, P. Wallace, and J. Olefsky. 1990. Use of a variable tracer infusion method to determine glucose turnover in man. Am. J. Physiol. 258:E16-E23.

13. Rowell, L. B., J. R. Blackmon, and R. A. Bruce. 1964. Indocyanine green clearance and estimated hepatic blood flow during mild to maximal exercise in upright man. J. Clin. Invest. 43:1677-1690.

14. Prager, R., P. Wallace, and J. M. Olefsky. 1986. In vivo kinetics of insulin action on peripheral glucose disposal and hepatic glucose output in normal and obese subjects. J. Clin. Invest. 78:472-481.

15. Ferrannini, E., J. Wahren, P. Felig, and R. A. DeFronzo. 1980. The role of fractional glucose extraction in the regulation of splanchnic glucose metabolism in normal and diabetic man. Metab. Clin. Exp. 29:28-35.

16. DeFronzo, R. A., E. Ferrannini, R. Hendler, P. Felig, and J. Wahren. 1983. Regulation of splanchnic and peripheral glucose uptake by insulin and hyperglycemia in man. Diabetes. 32:35-45.

17. Horwitz, D. L., J. I. Starr, M. E. Mako, W. G. Blackard, and A. H. Rubenstein. 1975. Proinsulin, insulin and C-peptide concentrations in human portal and peripheral blood. J. Clin. Invest. 55:1278-1283.

18. Stoll, R. W., J. L. Touber, L. A. Menahan, and R. H. Williams. 1970. Clearance of porcine insulin, proinsulin, and connecting peptide by the isolated rat liver. Proc. Soc. Exp. Biol. Med. 133:894-896.

19. Desbuquois, B., and G. D. Aurbach. 1971. Use of polyethylene glycol to separate free and antibody-bound peptide hormones in radioimmunoassays. $J$. Clin. Endocrinol. \& Metab. 33:732-738.

20. Faber, O. K., C. Binder, J. Markussen, L. G. Heding, V. K. Naithain, H. Kuzuya, B. Blix, D. L. Horwitz, and A. H. Rubenstein. 1978. Characterization of seven C-peptide sera. Diabetes. 27(Suppl. 1):170-177.

21. Unger, R., A. Eisenhart, M. McCall, and L. Madison. 1961. Glucagon antibodies and an immunoassay for glucagon. J. Clin. Invest. 48:1280-1289.

22. Gasic, S., C. H. Kleinbloesem, G. Heinz, and W. Waldhäusl. 1991. Contribution of splanchnic and peripheral vascular tissues to the disposal of angiotensinII and to regional conversion rates of angiotensin-I: a pilot study in humans. $J$. Cardiovasc. Pharmacol. 17:615-620.

23. Cherrington, A. D., M. J. Pagliasotti, S. R. Myers, B. Adkins-Marshall, and O. P. McGuinness. 1991. Factors which regulate net hepatic glucose uptake in vivo. J. Parenter. Enteral Nutr. 15:71s-73s.

24. Adkins, B. A., S. R. Myers, G. K. Hendrick, R. W. Stevenson, P. E. Williams, and A. D. Cherrington. 1987. Importance of the route of intravenous glucose delivery to hepatic glucose balance in the conscious dog. J. Clin. Invest. 79:557-565.

25. Adkins-Marshall, B. A., S. R. Myers, G. K. Hendrick, P. E. Williams, K. Triebwasser, B. Floyd, and A. D. Cherrington. 1989. Interaction between insulin and glucose-delivery route in regulation of net hepatic glucose uptake in conscious dogs. Diabetes. 39:87-95.

26. Cummins, A. J. 1952. Absorption of glucose and methionine from the human intestine: influence of the glucose concentration in the blood and the intestinal lumen. J. Clin. Invest. 31:928-937.

27. Radziuk, J. 1987. Tracer methods and the metabolic disposal of a carbohydrate load in man. Diabetes Metab. Rev. 3:231-267.

28. DeFronzo, R. A., E. Ferrannini, R. Hendler, J. Wahren, and P. Felig. 1978. Influence of hyperinsulinemia, hyperglycemia, and the route of glucose administration on splanchnic glucose exchange. Proc. Natl. Acad. Sci. USA. 75:5173-5177.

29. DeFronzo, R. A., E. Ferrannini, R. Hendler, P. Felig, and J. Wahren. 1983. Regulation of splanchnic and peripheral glucose uptake by insulin and hyperglycemia in man. Diabetes. 32:35-45.

30. Myers, S. R., O. P. McGuiness, D. W. Neal, and A. D. Cherrington. 1991. Intraportal glucose delivery alters the relationship between net hepatic glucose uptake and the insulin concentration. J. Clin. Invest. 87:930-939.

31. Consoli, A. 1992. Role of liver in pathophysiology of NIDDM. Diabetes Care. 15:430-441. 